In the article "Consumption of ultra-processed foods and socioeconomic position: a crosssectional analysis of the Brazilian Longitudinal Study of Adult Health", with DOI: 10.1590/0102311X000197, in the journal Cad Saúde Pública 2018; 34(3):e00019717.

doi: 10.1590/0102-311XER019717

The journal has been informed about some errors in the paper. The corrections are follows:

Where it reads:

\title{
Consumption of ultra-processed foods and socioeconomic position: a cross-sectional analysis of the Brazilian Longitudinal Study of Adult Health
}

O consumo de alimentos ultraprocessados e nível socioeconômico: uma análise transversal do Estudo Longitudinal de Saúde do Adulto, Brasil

Consumo de comida ultraprocesada y nivel socioeconómico: un análisis transversal del Estudio Longitudinal Brasileño sobre Salud en la Edad Adulta

It should read:

\section{Consumption of ultra-processed foods and socioeconomic position: a cross-sectional analysis of the Brazilian Longitudinal Study of Adult Health (ELSA-Brasil)}

Consumo de alimentos ultraprocessados e nível socioeconômico: uma análise transversal do Estudo Longitudinal de Saúde do Adulto (ELSA-Brasil)

Consumo de comida ultraprocesada y nivel socioeconómico: un análisis transversal del Estudio Longitudinal Brasileño sobre Salud en la Edad Adulta (ELSA-Brasil) 
Where it reads:

Bárbara dos Santos Simões 1

Letícia de Oliveira Cardoso 2

Isabela Judith Martins Benseñor 3

Maria Inês Schmidt 4

Bruce Bartholow Duncan 4

Vivian Cristine Luft 4

Maria del Carmen Bisi Molina 5

Sandhi Maria Barreto 1

Renata Bertazzi Levy ${ }^{3}$

Luana Giatti 1

\section{Correspondence}

B. S. Simões

Programa de Pós-graduação em Saúde Pública, Universidade Federal Minas Gerais.

Av. Alfredo Balena 190, Belo Horizonte, MG 30130-100, Brasil.

barbarassimoes@gmail.com

1 Programa de Pós-graduação em Saúde Pública, Universidade Federal Minas Gerais, Belo Horizonte, Brasil.

2 Escola Nacional de Saúde Pública Sergio Arouca, Fundação Oswaldo Cruz, Rio de Janeiro, Brasil.

3 Faculdade de Medicina, Universidade de São Paulo, São Paulo, Brasil.

4 Programa de Pós-graduação em Epidemiologia, Universidade Federal do Rio Grande do Sul, Porto Alegre, Brasil.

5 Programa de Pós-graduação em Saúde e Nutrição, Universidade Federal do Espírito Santo, Vitória, Brasil

It should read:

Bárbara dos Santos Simões 1

Sandhi Maria Barreto 1

Maria del Carmen Bisi Molina 2

Vivian Cristine Luft 3

Bruce Bartholow Duncan 3

Maria Inês Schmidt 3

Isabela Judith Martins Benseñor 4

Letícia de Oliveira Cardoso 5

Renata Bertazzi Levy 4

Luana Giatti 1

\section{Correspondence}

L. Giatti

Programa de Pós-graduação em Saúde Pública, Universidade Federal de Minas Gerais. Av. Alfredo Balena 190, Belo Horizonte, MG 30130-100, Brasil.

luana.giatti@gmail.com

1 Programa de Pós-graduação em Saúde Pública, Universidade Federal de Minas Gerais, Belo Horizonte, Brasil.

2 Programa de Pós-graduação em Saúde e Nutrição, Universidade Federal do Espírito Santo, Vitória, Brasil.

3 Programa de Pós-graduação em Epidemiologia, Universidade Federal do Rio Grande do Sul, Porto Alegre, Brasil.

4 Faculdade de Medicina, Universidade de São Paulo, São Paulo, Brasil.

${ }_{5}^{5}$ Escola Nacional de Saúde Pública Sergio Arouca, Fundação Oswaldo Cruz, Rio de Janeiro, Brasil. 
Where it reads:

We analyzed baseline data from the Brazilian Longitudinal Study of Adult Health (ELSA-Brasil 2008-2010; $N=14.378$ ) and data on dietary intake using a food frequency questionnaire, assigning it into three categories: unprocessed or minimally processed foods and processed culinary ingredients, processed foods, and ultra-processed foods.

It should read:

We analyzed baseline data from the Brazilian Longitudinal Study of Adult Health (ELSA-Brasil 2008-2010; $N=14,378$ ). Dietary intake, obtained through a food frequency questionnaire, was classified according extent and purpose of food processing in unprocessed or minimally processed foods and processed culinary ingredients, processed foods, and ultra-processed foods.

Where it reads:

Compared to individuals from upper income classes, the caloric contribution of ultra-processed foods was $10 \%, 15 \%$ and $20 \%$ lower among the ones from the three lowest income, respectively.

It should read:

Compared to individuals from upper income quintile, the caloric contribution of ultra-processed foods was 10\%, 15\% and 20\% lower among the ones from the three lowest income, respectively.

Where it reads:

O estudo teve como objetivo estimar a contribuição dos alimentos ultraprocessados à ingestão calórica total e investigar se essa contribuição difere de acordo com nível socioeconômico. Analisamos os dados da linha de base do Estudo Longitudinal de Saúde do Adulto-Brasil (ELSA-Brasil 2008-2010; $N=$ 14.378) e os de ingestão alimentar, usando um questionário sobre frequência de consumo alimentar, em três categorias: alimentos não processados ou minimamente processados e ingredientes culinários processados, alimentos processados e alimentos ultraprocessados.

It should read:

O estudo teve como objetivo estimar a contribuição dos alimentos ultraprocessados em relação ao consumo calórico total e investigar se essa contribuição difere de acordo com nível socioeconômico. Analisamos os dados da linha de base do Estudo Longitudinal de Saúde do Adulto-Brasil (ELSA-Brasil 20082010; $N$ =14.378). O consumo alimentar, obtido por questionário de frequência de consumo alimentar, foi classificado de acordo com o propósito e extensão do processamento em: alimentos não processados ou minimamente processados e ingredientes culinários processados, alimentos processados e alimentos ultraprocessados.

Where it reads:

Quando comparados aos indivíduos das classes de renda mais alta, a contribuição calórica dos ultraprocessados foi 10\%, 15\% e 20\% mais baixa entre aqueles pertencentes aos três quintis de renda mais baixos, respectivamente. 
It should read:

Quando comparados aos indivíduos do quintil de renda mais alta, a contribuição calórica dos ultraprocessados foi 10\%, 15\% e 20\% mais baixa entre aqueles pertencentes aos três quintis de renda mais baixos, respectivamente.

Where it reads:

El objetivo del estudio fue estimar la contribución de las comidas ultraprocesadas en la ingesta total calórica e investigar si difiere según el nivel socioeconómico. Analizamos datos de referencia, procedentes del Estudio Longitudinal Brasileño sobre Salud en la Edad Adulta (ELSA-Brasil 2008-2010; N = 14.378) $y$ datos de la ingesta nutricional, usando un cuestionario de frecuencia sobre comidas, asignándole tres categorías: comida sin procesar o mínimamente procesada e ingredientes culinarios procesados, comidas procesadas, y comidas ultraprocesadas.

It should read:

El objetivo del estudio fue estimar la contribución de las comidas ultraprocesadas en la ingesta total calórica e investigar si difiere según el nivel socioeconómico. Analizamos datos de referencia, procedentes del Estudio Longitudinal Brasileño sobre Salud en la Edad Adulta (ELSA-Brasil 2008-2010; $N=$ 14.378). El cosumo alimentario, obtenido por cuestionario de frecuencia sobre comidas, fue clasificado de acuerdo con el propósito y extensión de procesamiento en: comida sin procesar o mínimamente procesada e ingredientes culinarios procesados, comidas procesadas, $y$ comidas ultraprocesadas.

Where it reads:

Comparados con los individuos de las clases con ingresos superiores, la contribución calórica de las comidas ultraprocesadas fue un 10\%, 15\% y 20\% menor entre quienes pertenecían a las tres categorías de ingresos más bajas, respectivamente.

It should read:

Comparados con los individuos del quintil de renta más alta, la contribución calórica de las comidas ultraprocesadas fue un 10\%, 15\% y 20\% menor entre quienes pertenecían a las tres categorías de ingresos más bajas, respectivamente. 
Where it reads:

Table 5

Association between socioeconomic position indicators and caloric contribution percentage of ultra-processed foods. ELSA-Brasil (2008-2010).

Percentage caloric contribution of ultra-processed foods

AMR crude $(95 \% \mathrm{Cl})$

AMR adjusted * $(95 \% \mathrm{Cl})$

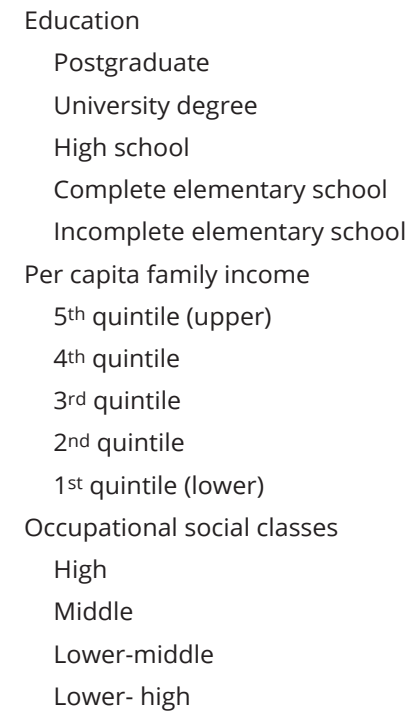

1.00

$0.98(0.96-1.00)$

$0.90(0.89-0.92)$ **

$0.82(0.80-0.84)$ **

$0.75(0.73-0.78)$ **

1.00

0.98 (0.96-0.99) ***

$0.94(0.91-0.96)$ **

$0.89(0.87-0.91)$ **

$0.83\left(0.82 \_0.85\right)$ **

1.00

0.98 (0.96_1.00)

$0.92(0.90-0.93)$ **

$0.89(0.87-0.92)$ **
1.00

$0.96(0.94-0.97)$ **

$0.88(0.87-0.90)$ **

$0.84(0.82-0.86)$ **

$0.80(0.77-0.82)$ **

1.00

$0.95(0.93-0.98)$ **

$0.90(0.88-0.92)$ **

$0.85(0.83-0.87)$ **

$0.80(0.79-0.83)$ **

1.00

$0.93(0.92-0.95) * *$

$0.88(0.86-0.90)$ **

$0.88(0.86-0.91) * *$

AMR: arithmetic mean ratios.

* Adjusted for gender and age;

** $\mathrm{p}<0.001$;

$* * * p<0.05$ 
It should read:

Table 5

Association between socioeconomic position indicators and caloric contribution percentage of ultra-processed foods. ELSA-Brasil (2008-2010).

\begin{tabular}{|c|c|c|}
\hline & \multicolumn{2}{|c|}{ Percentage caloric contribution of ultra-processed foods } \\
\hline & AMR crude $(95 \% \mathrm{Cl})$ & AMR adjusted * $(95 \% \mathrm{Cl})$ \\
\hline \multicolumn{3}{|l|}{ Education } \\
\hline Postgraduate & 1.00 & 1.00 \\
\hline University degree & $0.98(0.96-1.00)$ & $0.96(0.94-0.97) * *$ \\
\hline High school & $0.90(0.89-0.92) * *$ & $0.88(0.87-0.90) * *$ \\
\hline Complete elementary school & $0.82(0.80-0.84) * *$ & $0.84(0.82-0.86) * *$ \\
\hline Incomplete elementary school & $0.75(0.73-0.78) * *$ & $0.80(0.77-0.82) * *$ \\
\hline \multicolumn{3}{|l|}{ Per capita family income } \\
\hline $5^{\text {th }}$ quintile (upper) & 1.00 & 1.00 \\
\hline 4th quintile & $0.98(0.96-0.99) * * *$ & $0.95(0.93-0.98) * *$ \\
\hline 3rd quintile & $0.94(0.91-0.96) * *$ & $0.90(0.88-0.92) * *$ \\
\hline $2^{\text {nd }}$ quintile & $0.89(0.87-0.91) * *$ & $0.85(0.83-0.87) * *$ \\
\hline 1st quintile (lower) & $0.83\left(0.82 \_0.85\right) * \star$ & $0.80(0.79-0.83) * *$ \\
\hline \multicolumn{3}{|l|}{ Occupational social classes } \\
\hline High & 1.00 & 1.00 \\
\hline Middle & $0.98\left(0.96 \_1.00\right)$ & $0.93(0.92-0.95) * *$ \\
\hline Lower-middle & $0.92(0.90-0.93) * *$ & $0.88(0.86-0.90) * *$ \\
\hline Lower-high & $0.89(0.87-0.92)$ ** & $0.88(0.86-0.91)$ ** \\
\hline Lower-low & $0.83(0.82-0.85)$ ** & $0.83(0.81-0.85)$ ** \\
\hline
\end{tabular}

AMR: arithmetic mean ratios.

* Adjusted for gender and age;

$* * p<0.001$

$* * * p<0.05$. 